\title{
Can we improve the prediction of hip fracture by assessing bone structure using shape and appearance modelling?
}

S.R. Goodyear ${ }^{1}$, R.J.Barr ${ }^{1}$, E. McCloskey ${ }^{2}$, S. Alesci ${ }^{3}$, R.M. Aspden ${ }^{1}$, D.M.Reid ${ }^{1}$, J.S. Gregory ${ }^{1}$.

${ }^{1}$ Musculoskeletal Research Programme, Institute of Medical Sciences and Division of Applied Medicine, University of Aberdeen, Foresterhill, Aberdeen, AB25 2ZD, UK

${ }^{2}$ Academic Unit of Bone Metabolism and NIHR Musculoskeletal Biomedical Research Unit, University of Sheffield, Sheffield, S10 2RX, UK.

${ }^{3}$ Pfizer, 6 Dale Lane, Malvern, PA 19355, USA.

Correspondence to Dr J.S. Gregory, Research Fellow, IMS 2.23

University of Aberdeen

Foresterhill, ABERDEEN, AB25 2ZD,

United Kingdom.

j.gregory@abdn.ac.uk Tel: +44(0) 1224437966

Fax: +44(0) 1224437971 


\section{Abstract}

Purpose. There is a continuing need to improve the prediction of hip fractures to identify those at highest risk, enabling cost-effective use of preventative therapies.

Methods. The aim of this work was to validate an innovative imaging biomarker for hip fracture by modelling the shape and texture of the proximal femur assessed from dual energy X-ray absorptiometry (DXA) scans. Scans used were acquired at baseline from elderly patients participating in a prospective, placebo-controlled fracture prevention study of the bisphosphonate, clodronate. 182 subjects who subsequently suffered a hip fracture were age, weight and height matched with two controls who did not suffer a fracture during a median 4 year follow-up period. Logistic regression was used to test if variables were good predictors of fracture and adjust for bone mineral density (BMD).

Results. Shape mode 2, reflecting variability in neck-shaft angle, neck width and the size of both trochanters, $(0.81(\mathrm{OR}), 0.68-0.97(\mathrm{CI}), 0.024(P))$ and appearance mode 6 , recording grey-level contrast, $(1.33,1.11-1.59,0.002)$ were significant predictors of hip fracture and remained so after adjustment for BMD (shape mode $2(0.77,0.64-0.93,0.006)$, appearance mode $6(1.32,1.10-1.59,0.003))$. Receiver Operating Curve analysis showed the combination of shape mode 2, appearance mode 6 and BMD was $3 \%$ better than any single predictor.

Conclusion. Variables derived from shape and appearance models gave a prediction of fracture comparable to BMD and in combination with BMD gave an improvement in the prediction of hip fracture that could predict an additional 2000 hip fracture cases per year in the UK, potentially saving more than $£ 20$ million per year and 10,000 cases in the US. 


\section{Introduction and Background}

Approximately 340,000 hip fractures occur each year in the United States and, worldwide, the incidence of hip fractures is expected to more than triple from 1.66 million in 1990 to 6.26 million in 2050 [1]. In the European Union, an increase from 414 thousand to 972 thousand cases per annum is expected over the next 50 years [2]. In the UK a recent report from the National Hip Fracture Database, which excludes Scotland [3], has shown immediate management data on $76 \%$ of the estimated 70,000 cases occurring annually showing a 30 -day mortality rate of approximately $10 \%$. The cost of osteoporotic fractures to the UK economy has been estimated at $£ 1.7$ billion, of which more than a third is due to hip fracture [4]. The ability to identify individuals who are at very high risk of hip fracture would allow effective targeting of intervention strategies to substantially reduce the economic burden of fractures.

At present, the gold standard for the diagnosis of osteoporosis is bone mineral density (BMD) measurement by dual energy X-ray absorptiometry (DXA) [5], with each 1 standard deviation (SD) reduction in proximal femur BMD associated with an approximately 2.5- 2.8fold increased risk of fracture. However, BMD constitutes just one factor among many that contribute to the likelihood of an osteoporosis-related hip fracture and increasingly the additional risk factors are being captured using the FRAX risk algorithm [6]. The question remains whether further information on fracture risk can be gained by considering non-BMD information from the DXA scans currently used as the technique of choice to assess BMD. We have developed algorithms for the prediction of hip fracture risk using the shape and "texture" of the hip from traditional radiographic images, using active shape modelling (ASM) $[7,8]$. Subsequent work in progress has demonstrated that the ASM method can be applied to DXA scans [11-13]. Further, we have now developed an enhanced model of the femur using DXA images by using active appearance modelling (AAM) to capture the pattern of BMD intensities inside the femoral head and neck $[10,14,15,16]$. We found that 
many of the shape and the appearance modes were largely independent of total hip BMD, age and body mass index, indicating that the models may be useful for identifying a population of osteoporosis patients at very high risk of hip fracture. The current work sets out to test this hypothesis in a prospective study of incident hip fractures.

\section{Materials \& Methods}

\section{Study Cohort}

The data used for this study were baseline hip DXA (Hologic 4500) images taken from an MRC funded investigation into the effect of the bisphosphonate clodronate on fractures in almost 6000 women aged 75 years or older [17]. Ethical approval was given for the study by the local ethics review board. Whilst the principal aim of the study was to examine the efficacy of Clodronate, the study was also designed to investigate risk factors for fracture in elderly women, so the study group was designed to be a good representation of UK women of 75 and over. Women were recruited randomly from general practice lists, with no other inclusion criteria (e.g. existing osteoporosis). Exclusion criteria were concurrent treatment for a malignancy, concurrent medication likely to influence skeletal metabolism (other than calcium supplements 500 mg daily), bilateral hip arthroplasties, known malabsorptive states, impaired mental state or concurrent illness that would impede informed consent or compliance with the study, significant impairment of renal or hepatic function (serum creatinine $>300 \mathrm{M}$ or alanine aminotransaminase more than twice the upper limit of the reference range, respectively), and serum biochemistry consistent with underlying metabolic bone disease (e.g., osteomalacia) or calcium disorders other than primary hyperparathyroidism. 182 cases of incident hip fractures were documented during a median of 4 years follow-up. Baseline DXA hip images of women who went on to sustain a hip fracture were compared to those of 364 controls, matched for age, height and weight, who 
remained free of hip fracture during follow-up. Total hip BMD was measured using standard methods and has been reported previously [17].

\section{Active Shape and Appearance Modelling (ASM AND AAM)}

ASM and AAM were generated using the active appearance modelling toolkit software (Manchester University, UK) from points placed on each image describing the positions of particular anatomical features and the outline of the pelvis and femur as shown in Figure 1. The model had 72 points and the template design was derived from previous models developed in our group [18] and optimised to use features clearly and repeatedly visible in the Hologic scans used in this study. The AAM was designed so that it ignored non-bone areas, such as the space between the femoral head and acetabulum, in the analysis.

The modelling process is outlined in a flow diagram (Figure 2) In order to measure differences in the shape of a set of objects, they must first be aligned as closely as possible. The modelling software uses an affine-type transform (translation, rotation and scaling) to match the outline of the hips, without distorting the proportions. Principal component analysis (PCA) is then applied to produce a number of orthogonal modes that describe the variation of shape or appearance within the dataset. Modes are linear combinations of the original variables (point coordinates). The PCA process calculates the first mode to account for the largest amount of variance within the dataset, the second for the largest amount of variance still remaining and so on. As a result, the useful information in the dataset is contained in the first few modes that are retained for further analysis while the remainder can be discarded as noise. In this analysis the first 10 shape and appearance modes were examined, and mode scores were produced for each image. The modes are the output variables that describe the shape of the model that were analysed in this study. 
Mode scores were scaled and centred so the mean score and standard deviation for each mode were 0 and 1 respectively. All subsequent analysis was applied to the adjusted scores. No correction was made for the effect of clodronate treatment as only baseline images were used and the treatment had no impact on the incidence of hip fractures [17].

\section{Statistical Analysis}

Scores for the first 10 shape and appearance modes and BMD for the fracture and nonfracture groups were compared by $t$-test, where normally distributed (Shapiro-Wilk test) and logistic regression to determine which modes were useful for predicting fracture individually and when combined with other measures. Stepwise logistic regression was employed to determine the best set of variables for fracture prediction from the first 10 shape and appearance modes and BMD. Receiver operator characteristic (ROC) curves for each variable identified, and their combinations, were then compared to determine the additional benefit derived from including these variables in predicting hip fracture.

All statistical analysis was performed in SigmaPlot version 11.0 (Systat Software, Inc., Hounslow, UK) except for stepwise logistic regression which was performed in SPSS v20. Results are presented as mean (SD) unless otherwise stated.

\section{Results}

BMD values were significantly lower in the fracture group (Table 1). The mean BMD values translated to T-scores of $-2.36(1.09)$ and $-1.95(0.92)$ for the fracture and control groups respectively showing that the mean value of both groups was in the osteopenic but not the osteoporotic range.

When ASM mode scores for the fracture (F) and control (C) groups were compared by $t$-test modes 2 (F: -0.137 (1.006); C: 0.069 (0.991), P=0.023) and 4 (F: 0.171 (1.079); C: -0.086 
(0.948), $P=0.005)$ were significantly different, and mode 1 almost so (F: 0.105 (0.937); C: 0.052 (1.027), $P=0.083)$, while in the appearance model mode 6 (F: 0.185 (1.042); C: -0.092 (0.967), $P=0.002)$ was significantly different and mode 2 almost so (F: -0.115 (1.054); C: $0.057(0.968), P=0.057)$. Results are shown in Table 2 .

Logistic regression confirmed shape modes 2 and 4 and appearance mode 6 could be used to predict hip fracture (Table 3). When BMD was included in the model, these modes still made a significant contribution to the prediction equation, however appearance modes 2 and 3 also became significant predictors of fracture.

A forwards Wald stepwise logistic regression including the first 10 shape and appearance modes and BMD, selected shape mode 2 (Odds ratio $0.77,95 \% \mathrm{CI} 0.63-0.92, P=0.005$ ), appearance mode $6($ Odds ratio $1.33,95 \% \mathrm{CI} 1.10-1.61, P=0.003)$, and BMD as the best combination to predict fracture.

As shown in Figure 3, in shape mode 2 the fracture group (on average close to the light grey outline) had a narrower femoral neck with a larger neck-shaft angle. The acetabulum covered less of the head of the femur and both the greater and lesser trochanters were smaller.

As the appearance mode 6 mean scores were significantly greater in the fracture group than controls, images for the former group would generally appear to the left of Figure 4 and appear brighter with less contrast.

ROC curves comparing these variables (Figure 5) confirmed the combination of 3 variables (shape mode 2, appearance mode 6 and BMD) gave the best prediction of hip fracture, and BMD was the best single variable for fracture prediction. Statistical comparison of AUC between the ROC curves showed the improvement generated by adding shape mode 2 and appearance mode 6 to BMD was 3\%, but this did not reach significance. 


\section{Discussion/Conclusions}

Hip fractures remain a leading cause of morbidity and mortality globally among the elderly population. Their prevalence and economic burden is expected to increase exponentially over the next decades, which makes the development of reliable, cost-effective methods to improve their predictability a high priority in preventive medicine. While BMD remains a robust predictor of future risk for hip fracture, as also confirmed in our study, other parameters linked to bone quality may contribute significantly to determining such risk. The AAM has three groups of output variables, shape (as in the ASM model), texture (the BMD distribution) and appearance (a combination of both shape and texture). In a previous study of 465 women from the Aberdeen leg of the OPUS study [16], we tested these output variables for correlations with known risk factors for hip fracture. Finding that many of the shape and the appearance modes were independent of BMD, age and body mass index indicated that the models may provide additional predictive value for identifying a population of osteoporosis patients at very high risk of hip fracture.

In this study, shape and appearance models were built describing the variation in outline and grey-level variation in 546 baseline DXA images from the MRC clodronate study. Images from 182 individuals who went on to sustain a hip fracture were matched for age, height and weight but not BMD with twice that number of non-fracturing controls (Table 1). Because only baseline images were used there was no need to account for the use of clodronate and, in any case, clodronate was previously shown to have no effect on hip fracture [17].

Shape mode 2 showed that the neck-shaft angle was important in the prediction of hip fracture, with a larger angle being found in the fracture group. A bigger neck-shaft angle has generally been observed in fracture groups in both men and women [19-24]. It has also been 
associated with reduced bone strength [25,26]. An advantage of shape modelling is that the modes of variation identify geometrical features that tend to vary together and, in this case, shape mode 2 included the area of the femoral head covered by the acetabulum (less in hips that fractured) and the size of the greater and lesser trochanters as well as neck-shaft angle.

While appearance mode 6 scores showed significant predictive capacity, it is perhaps surprising that, in those who went on to sustain a fracture, images had less variation in BMD between cortical and trabecular or bone marrow regions than controls. The images used in this study, however, were not true BMD maps. The image grey-level has some relationship to BMD known to Hologic (the DXA machine manufacturer) but this was not shared with us because of Intellectual Property issues between Hologic and the study funder. As a result there is an unknown amount of scan to scan variation, unrelated to the patients' BMD. This will not affect the shape data but the effects on the image intensity and contrast will introduce an additional level of noise to the appearance model. This may also affect the predictive capacity and partly explain why this was poorer than our previous study in which the combination of BMD, ASM and trabecular structure showed almost complete separation of 50 matched hip fracture and control cases as shown by ROC analysis [10].

In that previous cross-sectional study, the discrimination of hip fracture cases from controls using BMD measurements was considerably improved by the addition of shape information and a measure of trabecular architecture, using Fourier transforms, from radiographs. The area under the ROC curve was higher for shape models (0.81), and for femoral neck BMD (0.79) than in the prospective study reported here (shape: 0.57, BMD: 0.62). These lower values are likely due to the participants in the current study being older (81.5 against 69.2 years), better matched with controls and measurements being made prospectively. We formerly used images from the contralateral hip collected after fracture and Reider et al have shown that patients who had had a hip fracture lost BMD in their unfractured hips in the 12 
months following fracture [27], thereby magnifying differences between fracture and control groups.

The trends found in this and the previous study are similar however, and when shape and appearance models are added to BMD the area under the ROC curve increased to 0.65 just as the combined scores gave increased prediction in the previous study (AUC: 0.95). This is a similar increase to a study recently published by Whitmarsh et al. who compared shape and BMD distribution from DXA scans using a model built using CT scans. They found adding the model data increased the area under the curve by $4 \%$ (from 0.80 for BMD alone to 0.84 ). \{ $\{19499$ Whitmarsh,T. 2012 $\}\}$ In a more recent study in radiographs, Baker-LePain et al found shape modes had an AUC of 0.81 compared to BMD areas of 0.68 (femoral neck) or 0.65 (intertrochanter), and in a combined model the AUC increased to 0.84 [28]. In that study, there was no matching between fractures and controls and the follow up period (mean 8.3 years) was almost double that of our study.

One of the limitations of this study is that we analysed images from only one Hologic scanner. While this ensured that all the images were directly comparable, it limits the generalization of the approach. We would expect that the ASM/AAM approach could be used in other scanners. To explore this in detail, however, studies using a mixture different scanners will be required. The population is another limiting factor. As with any study, the inclusion and exclusion criteria place limits on the generalizability of the findings to other patient groups. The study was limited to women of 75 years or older and had relatively few exclusion criteria, as it was designed to determine fracture risk factors, as well as to test the efficacy of Clodronate. In particular, all women who were already on medications likely to 
alter their skeletal metabolism (barring calcium supplements), or whose serum biochemistry indicated metabolic bone disease were automatically excluded from the study.

Identifying hip shape and appearance as risk factors raises the question of whether they can be modified to provide protection against fracture. Studies of hip axis length (HAL) and other geometrical measures may help to provide some clues. Between $51 \%$ and $79 \%$ of hip axis length is genetically determined [6,7], which leaves between $21 \%$ and $49 \%$ as a potentially modifiable factor. Whilst adult HAL is reached by the mid teens (Goulding et al 1996; Flicker et al 1996), femoral geometry does not remain stable throughout adult life. Longitudinal studies have found that the shape of the femur continues to change, with both the shaft and neck width increasing with age (Heaney et al 1997; Beck et al 2000). Some modifiable factors such as weight and physical activity are significantly linked to geometrical measures ( Yang et al 1999; Gnudi et al 1999; Nikander et al 2012 Heinonen et al 2012), but the largest changes are likely to be seen from pharmaceutical interventions. Strontium Ranelate in particular is likely to change the appearance model as the strontium is absorbed preferentially over calcium and results in marked increases in BMD. However no studies have yet investigated this change over time.

Our study showed that including shape and appearance modes in the prediction of hip fracture added $3 \%$ to the number of fractures correctly predicted. Although this appears to be a small number, and it did not reach statistical significance at the 0.05 level, its potential clinical significance is considerable. Given the large and growing number of hip fractures occurring annually, in Europe [2] and across the world [1] and their massive cost to society [4], the absolute benefit is worth further consideration. At the $3 \%$ level, more than 2000 additional hip fractures, costing the British economy more than $£ 20$ million, could be predicted, alongside an additional 10,000 in the US. 
BMD measurement is fully integrated into most DXA scanners, and results are generated with a minimum of supervision - a radiographer should verify the measurement regions do not overlap extra bony regions. To be genuinely considered for addition to a fracture prediction algorithm, shape and appearance modelling would need to demand the same or less supervision. While unsupervised point placement is not currently available, Roberts et al have demonstrated effective, automatic point placement when shape modelling vertebrae in the spine in X-ray and DXA images [29,30].

Shape and appearance models show great promise for the prediction of future osteoarthritis in baseline DXA images [31], and an automatic, combined screening tool deployed in DXA scanners would make a useful addition to the rheumatologist's armamentarium.

In conclusion, this study shows that shape and appearance information from DXA images is equally effective as BMD alone in the prediction of hip fractures, and remains a good predictor of hip fracture after adjustment for BMD. While the percentage increase in hip fracture prediction gained on adding shape and appearance variables to the prediction algorithm is modest, in absolute terms this represents the prediction of about 2000 additional hip fractures per year in the UK that cost the economy more than $£ 20$ million. This also corresponds to over 10,000 hip fracture cases in the US and, based on projections [1] over 180,000 worldwide. Previous studies using radiographs $[10,28]$ have shown shape and appearance models add significantly to the prediction of hip fracture, suggesting further studies in DXA images should be conducted to confirm our current findings. 


\section{Acknowledgements}

This work was supported by an award (WHMSB-AU-115) from the Translational Medicine Research Collaboration - a consortium made up of the Universities of Aberdeen, Dundee, Edinburgh and Glasgow, the four associated NHS Health Boards (Grampian, Tayside, Lothian and Greater Glasgow \& Clyde), Scottish Enterprise and Pfizer (formerly Wyeth).

JSG is supported by the Medical Research Council [G0901242].

\section{Disclosures}

No disclosures 


\section{References}

[1] Cooper C., G. Campion, L.J. Melton III, Hip fractures in the elderly: A world-wide projection, Osteoporosis Int. 2 (1992) 285-9.

[2] Walker-Bone K., E. Dennison, C. Cooper, Epidemiology of osteoporosis, Rheumatic Disease Clinics of North America. 27 (2001) 1-18.

[3] Currie C., M. Partridge, F. Plant, J. Roberts, R. Wakeman, A. Williams, The national hip fracture database national report 2011 (2011). http://www.nhfd.co.uk/003/hipfractureR.nsf/0/81d394d97ddba0e9802578cc002c12d4/\$FILE/ NHFD\%20National\%20Report\%202011.pdf

[4] Holroyd C., C. Cooper, E. Dennison, Epidemiology of osteoporosis, Best Practice and Research: Clinical Endocrinology and Metabolism. 22 (2008) 671-85.

[5] Blake G.M., I. Fogelman, Role of dual-energy X-ray absorptiometry in the diagnosis and treatment of osteoporosis, Journal of Clinical Densitometry. 10 (2007) 102-10.

[6] Kanis J.A., A. Oden, H. Johansson, F. Borgström, O. Ström, E. McCloskey, FRAX® and its applications to clinical practice, Bone. 44 (2009) 734-43.

[7] Cootes T.F., C.J. Taylor, D.H. Cooper, J. Graham, Active shape models-their training and application, Comput. Vision Image Understanding. 61 (1995) 38-59.

[8] Gregory J.S., D. Testi, A. Stewart, P.E. Undrill, D.M. Reid, R.M. Aspden, A method for assessment of the shape of the proximal femur and its relationship to osteoporotic hip fracture, Osteoporosis International. 15 (2004) 5-11. 
[9] Gregory J.S., A. Stewart, P.E. Undrill, D.M. Reid, R.M. Aspden, Identification of hip fracture patients from radiographs using fourier analysis of the trabecular structure: A crosssectional study, BMC Medical Imaging. 4 (2004) 4.

[10] Gregory J.S., A. Stewart, P.E. Undrill, D.M. Reid, R.M. Aspden, Bone shape, structure, and density as determinants of osteoporotic hip fracture: A pilot study investigating the combination of risk factors, Investigative Radiology. 40 (2005) 591-7.

[11] Gregory J.S., A. Stewart, D.M. Reid, R.M. Aspden, Repeatability of active shape modelling of the proximal femur for radiographs and DXA scans, J. Bone Miner. Res. 20 (2005) P43.

[12] Waarsing J.H., R.M. Rozendaal, J.A.N. Verhaar, S.M.A. Bierma-Zeinstra, H. Weinans, A statistical model of shape and density of the proximal femur in relation to radiological and clinical OA of the hip, Osteoarthritis and Cartilage. 18 (2010) 787-94.

[13] Roberts M., T.F. Cootes, J.E. Adams, Vertebral morphometry: Semiautomatic determination of detailed shape from dual-energy X-ray absorptiometry images using active appearance models, Invest. Radiol. 41 (2006) 849-59.

[14] Cootes T.F., C.J. Taylor, Statistical models of appearance for medical image analysis and computer vision, Proceedings of SPIE - The International Society for Optical Engineering. 4322 (2001) 236-48.

[15] Edwards G.J., T.F. Cootes, C.J. Taylor, Advances in active appearance models, Proceedings of the IEEE International Conference on Computer Vision. 1 (1999) 137-42. 
[16] Gregory J.S., A. Stewart, D.M. Reid, R.M. Aspden, Bone shape, texture and appearance from DXA scans and the relationship with age, body mass index and bone mineral density: An analysis from the OPUS study, Osteoporosis International. 17 (2006) 370.

[17] McCloskey E.V., M. Beneton, D. Charlesworth, K. Kayan, D. DeTakats, A. Dey, J. Orgee, R. Ashford, M. Forster, J. Cliffe, L. Kersh, J. Brazier, J. Nichol, S. Aropuu, T. Jalava, J.A. Kanis, Clodronate reduces the incidence of fractures in community-dwelling elderly women unselected for osteoporosis: Results of a double-blind, placebo-controlled randomized study, Journal of Bone and Mineral Research. 22 (2007) 135-41.

[18] Gregory J.S., R.J. Barr, K. Yoshida, D.M. Reid, A.J. Silman, G. Hosie, S. Alesci, G.J. Macfarlane, R.M. Aspden, Nested shape models for medical image analysis: Call for consensus on hip shape modeling, Osteoarthritis and Cartilage. 16 (2008) S161.

[19] Gómez Alonso C., M. Díaz Curiel, F. Hawkins Carranza, R. Pérez Cano, A. Díez Pérez, Femoral bone mineral density, neck-shaft angle and mean femoral neck width as predictors of hip fracture in men and women, Osteoporosis Int. 11 (2000) 714-20.

[20] Karlsson K.M., I. Sernbo, K.J. Obrant, I. Redlund-Johnell, O. Johnell, Femoral neck geometry and radiographic signs of osteoporosis as predictors of hip fracture, Bone. 18 (1996) 327-30.

[21] Partanen J., T. Jämsä, P. Jalovaara, Influence of the upper femur and pelvic geometry on the risk and type of hip fractures, Journal of Bone and Mineral Research. 16 (2001) 1540-6.

[22] Gnudi S., C. Ripamonti, L. Lisi, M. Fini, R. Giardino, G. Giavaresi, Proximal femur geometry to detect and distinguish femoral neck fractures from trochanteric fractures in postmenopausal women, Osteoporosis Int. 13 (2002) 69-73. 
[23] Boonen S., R. Koutri, J. Dequeker, J. Aerssens, G. Lowet, J. Nijs, G. Verbeke, E. Lesaffre, P. Geusens, Measurement of femoral geometry in type I and type II osteoporosis: Differences in hip axis length consistent with heterogeneity in the pathogenesis of osteoporotic fractures, Journal of Bone and Mineral Research. 10 (1995) 1908-12.

[24] Gnudi S., C. Ripamonti, G. Gualtieri, N. Malavolta, Geometry of proximal femur in the prediction of hip fracture in osteoporotic women, Br. J. Radiol. 72 (1999) 729-33.

[25] Pulkkinen P., F. Eckstein, E.-. Lochmüller, V. Kuhn, T. Jämsä, Association of geometric factors and failure load level with the distribution of cervical vs. trochanteric hip fractures, Journal of Bone and Mineral Research. 21 (2006) 895-901.

[26] Kukla C., C. Gaebler, R.W. Pichl, R. Prokesch, G. Heinze, T. Heinz, Predictive geometric factors in a standardized model of femoral neck fracture: Experimental study of cadaveric human femurs, Injury. 33 (2002) 427-33.

[27] Reider L., T.J. Beck, M.C. Hochberg, W.G. Hawkes, D. Orwig, J.A. Yuyahiro, J.R. Hebel, J. Magaziner, Women with hip fracture experience greater loss of geometric strength in the contralateral hip during the year following fracture than age-matched controls, Osteoporosis Int. 21 (2010) 741-50.

[28] Baker-Lepain J.C., K.R. Luker, J.A. Lynch, N. Parimi, M.C. Nevitt, N.E. Lane, Active shape modeling of the hip in the prediction of incident hip fracture, Journal of Bone and Mineral Research. 26 (2011) 468-74.

[29] Roberts M.G., T. Oh, E.M.B. Pacheco, R. Mohankumar, T.F. Cootes, J.E. Adams, Semiautomatic determination of detailed vertebral shape from lumbar radiographs using active appearance models, Osteoporosis Int. (2011) 1-10. 
[30] Roberts M.G., E.M.B. Pacheco, R. Mohankumar, T.F. Cootes, J.E. Adams, Detection of vertebral fractures in DXA VFA images using statistical models of appearance and a semiautomatic segmentation, Osteoporosis Int. 21 (2010) 2037-46.

[31] Gregory J.S., J.H. Waarsing, J. Day, H.A. Pols, M. Reijman, H. Weinans, R.M. Aspden, Early identification of radiographic osteoarthritis of the hip using an active shape model to quantify changes in bone morphometric features: Can hip shape tell us anything about the progression of osteoarthritis?, Arthritis Rheum. 56 (2007) 3634-43. 
Table 1 The study population characteristics Values shown as mean (standard deviation) and $P$ values are from $t$ test.

\begin{tabular}{c|cc|cc|c} 
& \multicolumn{2}{|c|}{ Fracture } & \multicolumn{2}{|c|}{ Non Fracture } & $P$ \\
\hline Age yrs & 81.5 & $(4.6)$ & 81.5 & $(4.5)$ & 0.96 \\
HEIGHT cm & 154.9 & $(6.1)$ & 154.9 & $(6.0)$ & 0.97 \\
WEIGHT kg & 59 & $(10)$ & 59 & $(10)$ & 0.88 \\
BMD g/cm & 0.66 & $(0.13)$ & 0.70 & $(0.11)$ & $<0.001$
\end{tabular}

Table $2 P$ values from a comparison between fracture and non-fracture group scores from the shape and appearance models. Significantly different modes $(P<0.05)$ are marked in bold and modes of interest $(P<0.01)$ are in italics. Modes marked with $\dagger$ were analysed using Mann-Whitney tests, all other modes were marked with $t$-tests.

\begin{tabular}{c|c|c} 
mode & shape & appearance \\
\hline 1 & 0.083 & 0.15 \\
2 & $\mathbf{0 . 0 2 3}$ & $0.067^{\dagger}$ \\
3 & 0.16 & 0.17 \\
4 & $\mathbf{0 . 0 0 5}$ & $0.26^{\dagger}$ \\
5 & 0.16 & $0.69^{\dagger}$ \\
6 & 0.26 & $\mathbf{0 . 0 0 2}$ \\
7 & $0.71^{\dagger}$ & 0.60 \\
8 & 0.42 & 0.99 \\
9 & 0.38 & 0.31 \\
10 & 0.76 & 0.79
\end{tabular}

Table 3 Logistic regression assessing shape and appearance modes useful in predicting fracture and the affect of adjusting for BMD.

\begin{tabular}{c|ccc} 
Variable & $\begin{array}{l}\text { Odds } \\
\text { Ratio }\end{array}$ & $\mathbf{9 5 \%}$ Cl & $\begin{array}{c}\boldsymbol{P} \\
\text { value }\end{array}$ \\
\hline \multicolumn{4}{|c}{ Unadjusted } \\
\hline Sh Mode 1 & 1.17 & $0.98-1.40$ & 0.084 \\
Sh Mode 2 & 0.81 & $0.68-0.97$ & $\mathbf{0 . 0 2 4}$ \\
Sh Mode 4 & 1.30 & $1.08-1.55$ & $\mathbf{0 . 0 0 5}$ \\
\hline Ap Mode 2 & 0.84 & $0.71-1.01$ & 0.058 \\
Ap Mode 3 & 1.14 & $0.95-1.36$ & 0.17 \\
Ap Mode 6 & 1.33 & $1.11-1.59$ & $\mathbf{0 . 0 0 2}$ \\
\hline BMD & 0.033 & $0.0073-0.15$ & $<\mathbf{0 . 0 0 1}$ \\
\hline \multicolumn{4}{c}{ Adjusted for BMD } \\
\hline Sh Mode 1 & 1.16 & $0.96-1.39$ \\
Sh Mode 2 & 0.77 & $0.64-0.93$ & 0.12 \\
Sh Mode 4 & 1.28 & $1.06-1.53$ & $\mathbf{0 . 0 1 0}$ \\
\hline Ap Mode 2 & 0.83 & $0.69-0.99$ & $\mathbf{0 . 0 3 8}$ \\
Ap Mode 3 & 1.21 & $1-1.45$ & $\mathbf{0 . 0 4 8}$ \\
Ap Mode 6 & 1.32 & $1.1-1.59$ & $\mathbf{0 . 0 0 3}$
\end{tabular}




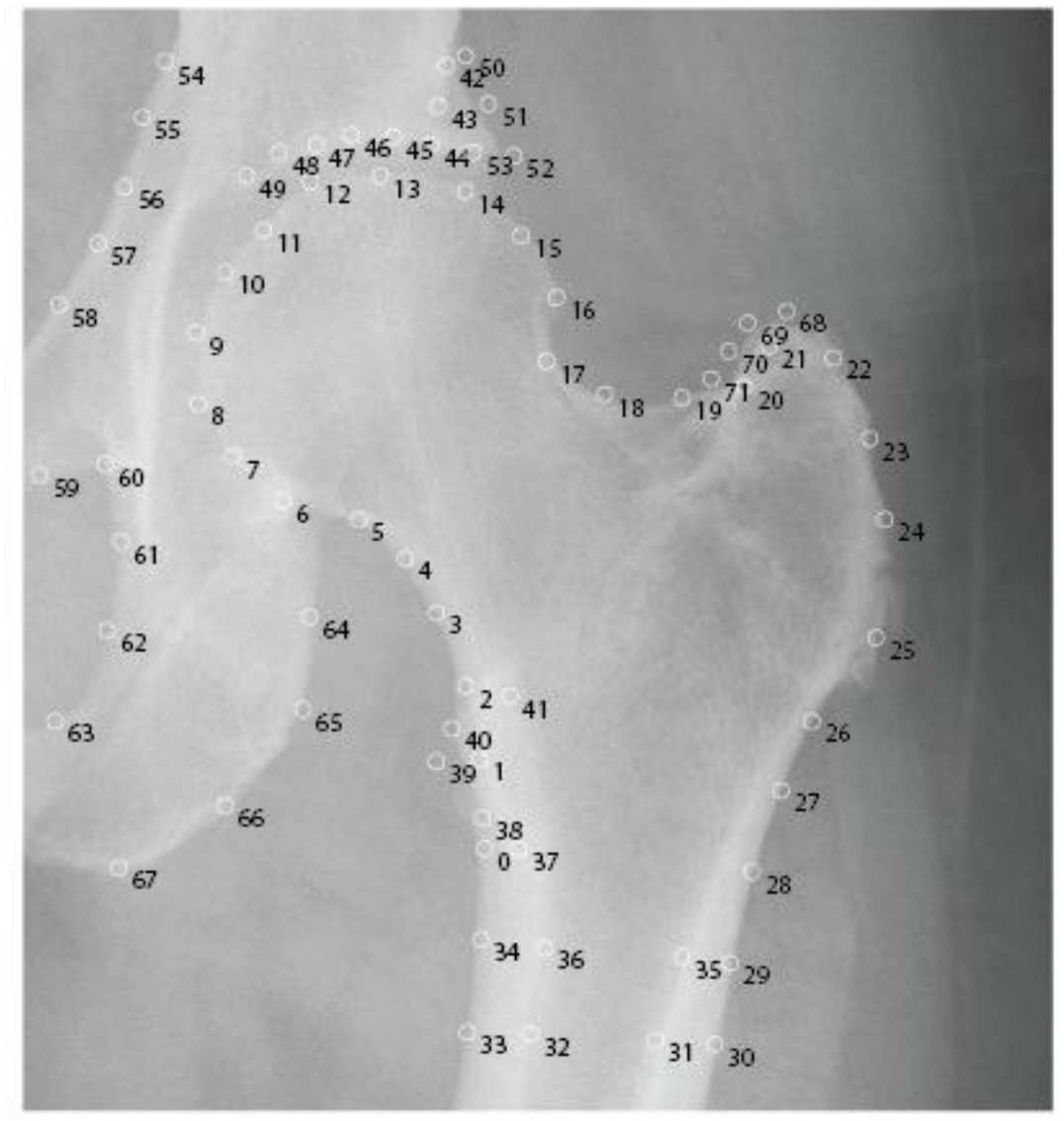

Figure 1. The point positions for the 72 point model, outlining the femur and parts of the pelvis. 


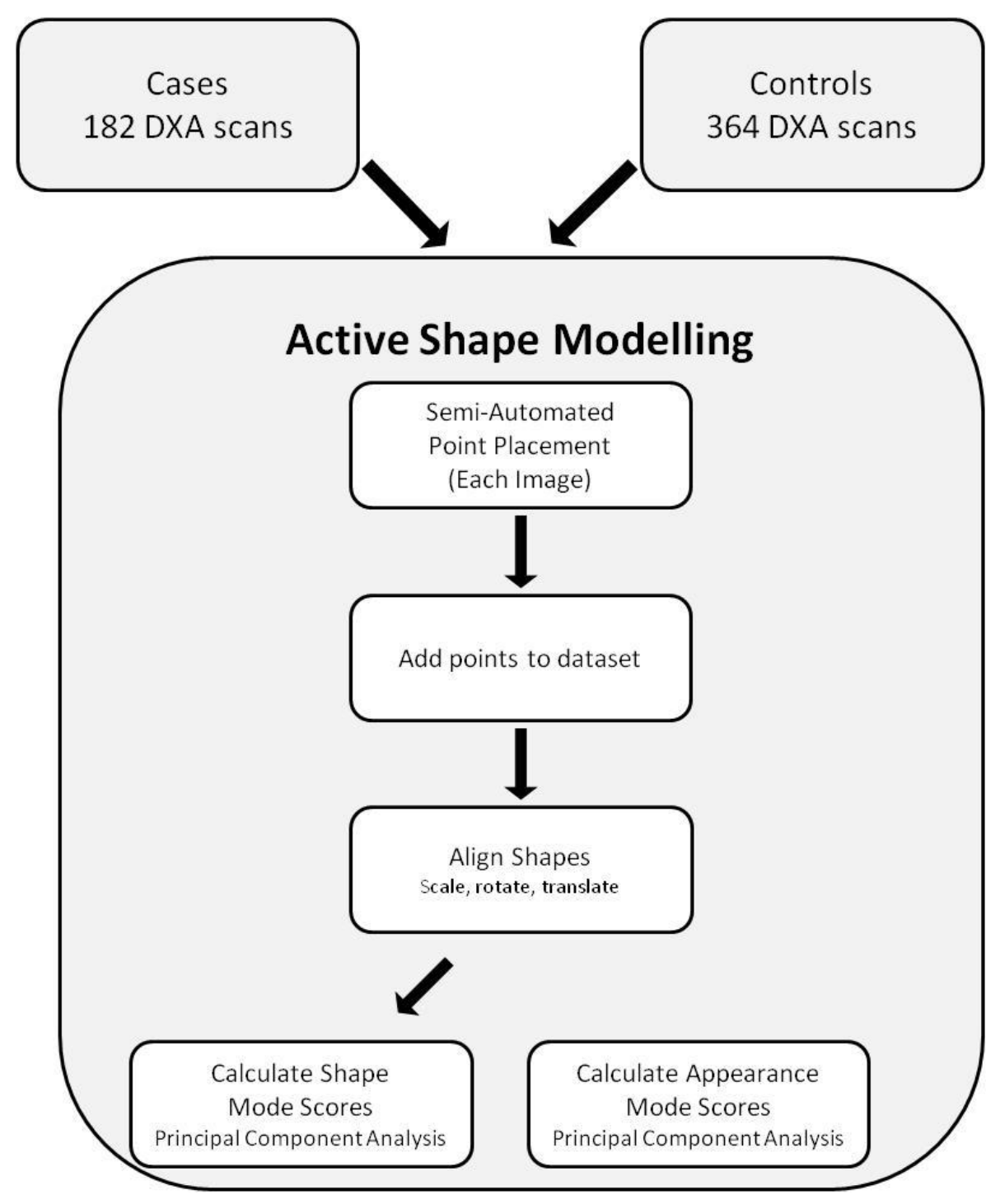

Figure 2. A flow-diagram showing the steps of the modeling process. 


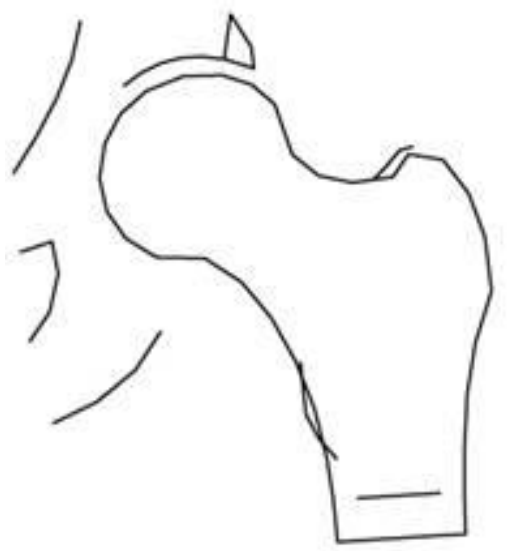

-2 st. dev (high risk)

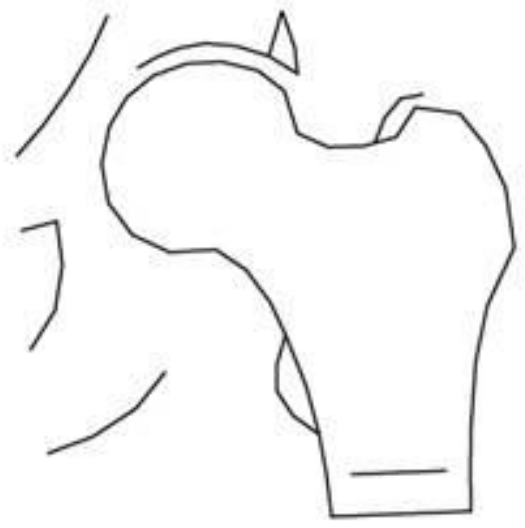

+2 st. dev (low risk)

Figure 3 The variation in shape mode 2. On average, hips that went on to fracture were similar to the light grey outline and had a greater neck-shaft angle, smaller trochanters and a smaller acetabular coverage. 


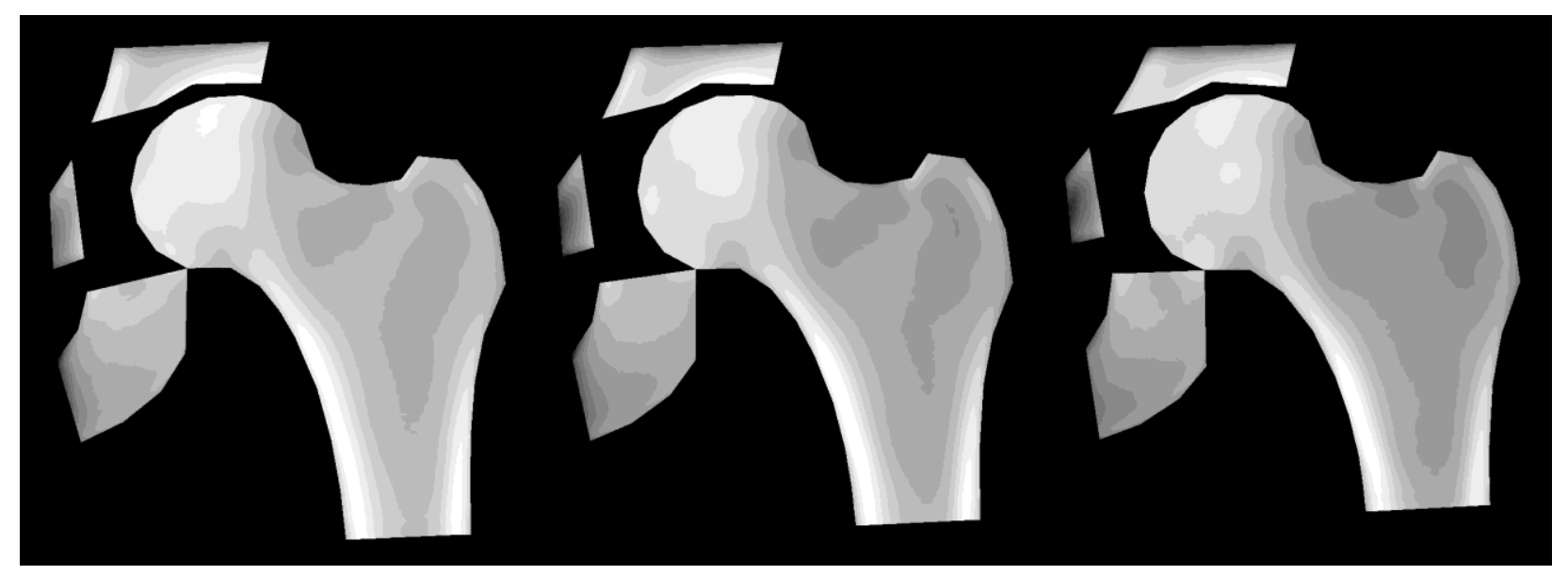

Figure 4 Appearance model mode 6 . Here the fracture group is represented by the left-hand image that shows less variation in grey-scale across the image. 


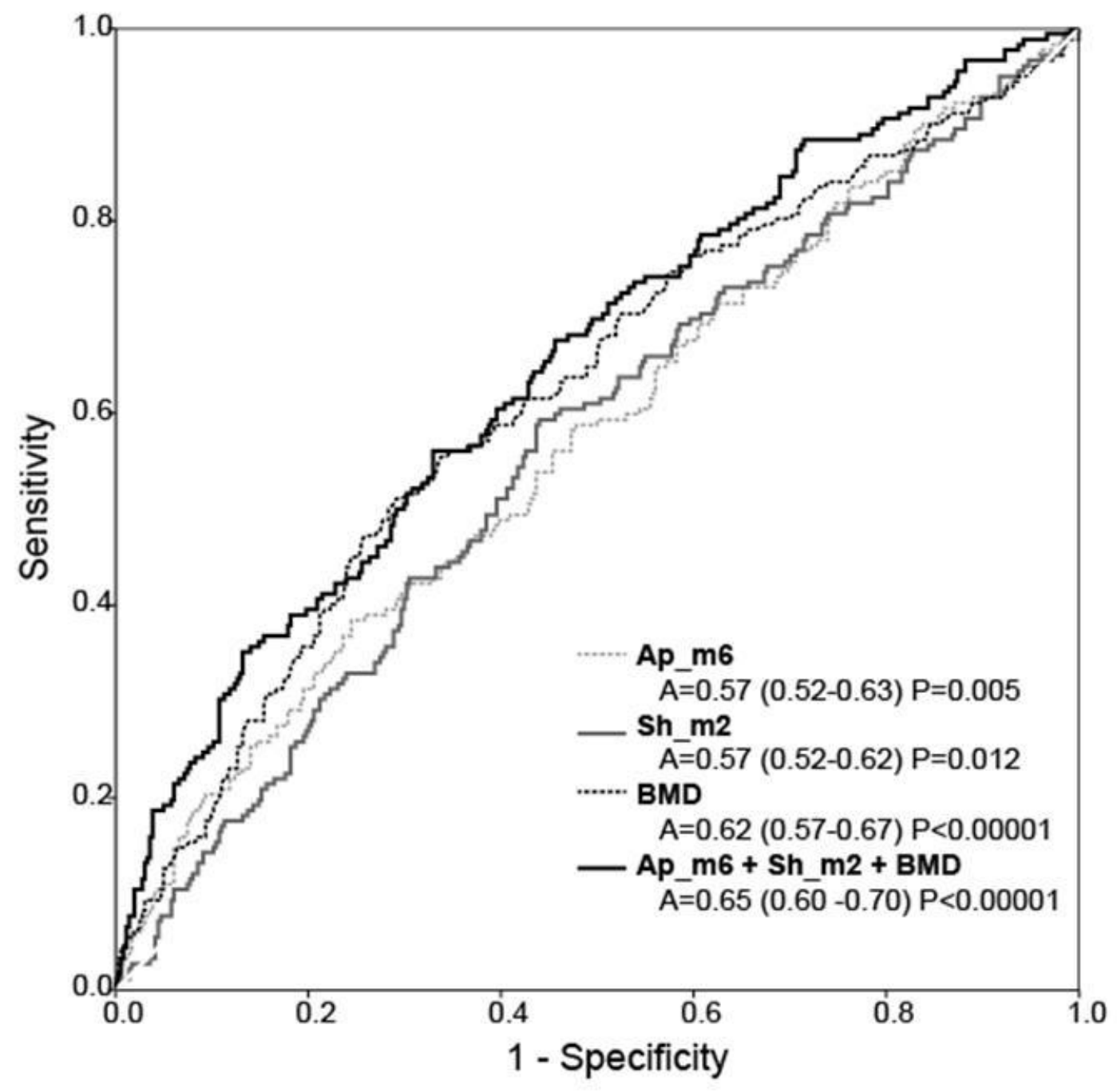

\begin{tabular}{l|ll} 
Variables & $\begin{array}{l}\text { Difference in Area } \\
\text { compared to BMD }\end{array}$ & $\begin{array}{l}\mathbf{P} \\
\text { value }\end{array}$ \\
\hline Sh_m2 & 0.054 & 0.16 \\
Ap_m6 & 0.046 & 0.20 \\
Apm6+ Sh_m2 + BMD & 0.028 & 0.13
\end{tabular}

Figure 5 ROC analysis. Curves showing shape mode 2 and appearance mode 6 as predictors of fracture. The combination of BMD and these modes gave the best prediction of hip fracture, measured by area under the curve, but the improvement in prediction over BMD alone was not statistically significant. 